\title{
Immunotherapy is Associated with a Survival Benefit in Patients Receiving Chemotherapy for Metastatic Pancreas Cancer
}

\author{
Jonathan J. Hue
}

UH CMC: UH Cleveland Medical Center https://orcid.org/0000-0002-1608-8874

\section{Katherine Bingmer}

UH CMC: UH Cleveland Medical Center

\section{Kavin Sugumar}

UH CMC: UH Cleveland Medical Center

\section{Sarah C. Markt}

Case Western Reserve University School of Medicine

\section{Luke D. Rothermel}

UH CMC: UH Cleveland Medical Center

Jeffrey M. Hardacre

UH CMC: UH Cleveland Medical Center

John B. Ammori

UH CMC: UH Cleveland Medical Center

Jordan M. Winter

UH CMC: UH Cleveland Medical Center

Lee M. Ocuin ( $\square$ lee.ocuinmd@gmail.com )

Division of Hepatobiliary and Pancreatic Surgery

\section{Research article}

Keywords: pancreatic neoplasm, immunotherapy, chemotherapy, survival analysis

Posted Date: December 7th, 2020

DOl: https://doi.org/10.21203/rs.3.rs-121110/v1

License: (c) (i) This work is licensed under a Creative Commons Attribution 4.0 International License. Read Full License 


\section{Abstract}

Background: Immunotherapy (IT) has led to improved survival in several common cancers but success in pancreatic ductal adenocarcinoma (PDAC) has been limited. We analyzed if combination ITchemotherapy (IT-CT) is associated with improved survival compared to chemotherapy alone (CT) in patients with metastatic PDAC.

Methods: The National Cancer Database (2004-2016) was queried for patients who were diagnosed with metastatic PDAC. Patients were categorized by treatment group: CT only and IT-CT. Patients were excluded if they received radiation or a surgical procedure. The primary outcome was overall survival.

Results: A total of 59,289 patients were identified, of whom 58,947 (99.4\%) received CT and $342(0.6 \%)$ received IT-CT. The IT-CT group was younger, had fewer comorbidities, and was more often treated at an academic center. The utilization of multi-agent chemotherapy was similar between the groups. Median survival of patients treated with IT-CT was longer than CT alone (7.9 vs 6.3 months, $p=0.005)$. On multivariable analysis, receipt of IT-CT was associated with a survival advantage as compared to CT $(\mathrm{HR}=0.86,95 \% \mathrm{Cl} 0.76-0.97)$ when adjusting for demographics and type of chemotherapy regimen.

Conclusions: In patients with metastatic PDAC, it appears that combination IT-CT may be associated with a survival advantage compared to CT alone.

\section{Background}

Multi-agent chemotherapy remains the backbone of systemic treatment for patients with pancreatic ductal adenocarcinoma (PDAC).[1] FOLFIRINOX (5-fluorouracil, leucovorin, irinotecan, oxaliplatin), gemcitabine with nab-paclitaxel, and gemcitabine with capecitabine have demonstrated a survival benefit over gemcitabine monotherapy in patients with metastatic PDAC.[2-4] Despite modest advances in the effectiveness of chemotherapeutics, further combinations are unlikely. Multi-agent chemotherapy is associated with severe toxicities, which have been reported in the majority of patients receiving FOLFIRINOX.[2, 5] Alternative treatment strategies are needed. There has been improvement in overall survival of other solid tumor malignancies over the past decade, such as melanoma, renal cell carcinoma, lung cancer, and bladder cancer, with the introduction of therapy targeting various immune system checkpoints.[6-9] Immunotherapy aims to boost a patient's immune system to enhance tumor recognition and destruction.[10-14] Unfortunately, no such success has been realized in the treatment of PDAC to date.[15-21]

A previous retrospective analysis of patients with PDAC who underwent a pancreatectomy in the National Cancer Database (NCDB) demonstrated an association between improved overall survival and adjuvant immunotherapy-chemotherapy as compared to adjuvant chemotherapy alone.[22] To our knowledge, there has not been a report on the impact of immunotherapy in metastatic PDAC patients using a large administrative database. The primary aim of this study was to use the NCDB to determine if the addition of immunotherapy to chemotherapy was associated with a survival advantage in patients with 
metastatic PDAC. In addition, we aimed to identify potential disparities in receipt of immunotherapy based on sociodemographic characteristics.

\section{Methods}

Institutional assurances. The Institutional Review Boards at our institutions have deemed that retrospective analyses of public, anonymized data sets are exempt from review.

Data source. The NCDB is a nationwide database, jointly maintained by the American College of Surgeons and the American Cancer Society. This database receives patient information from Commission on Cancer-accredited hospitals across the nation and contains de-identified information on approximately $70 \%$ of all new malignancies diagnosed in the United States each year. The accuracy of the data reported, statistical analyses performed, and conclusions drawn are not monitored nor verified by the American College of Surgeons or American Cancer Society. The NCDB Participant User File data dictionary contains definitions of variables used in this study.[23]

Patient cohort. Adult patients diagnosed with PDAC were identified between 2004 and 2016, using International Classification for Diseases in Oncology (3rd Edition) codes 8140 and 8500. Only patients with metastatic (stage IV) disease at diagnosis were included. Patients were excluded from the analysis if they underwent a surgical procedure, if they received radiotherapy, if the type of chemotherapy administered (single-agent or multi-agent) was not recorded, or if data on immunotherapy administration was missing (consort diagram, Fig. 1). For this analysis, we utilized data on sociodemographic (age, sex, race, insurance status, education quartile, income quartile, Charlson-Deyo comorbidity index) and clinical characteristics, facility type (academic, community, comprehensive community, integrated), and treatment information.

Exposure. Patients with PDAC were categorized into two treatment groups: those who received single- or multi-agent chemotherapy (CT) alone or immunotherapy (IT) and CT (IT-CT).

Outcomes. The primary outcome in this study was overall survival, defined as the number of months from the patient's date of diagnosis to either their date of death or last follow-up. Cancer-specific outcomes were not evaluated because the NCDB does not capture data pertaining to recurrence or cause of death.

Statistical analysis. Clinical and demographic data were compared between the treatment groups; the Wilcoxon rank-sum test was used to compare continuous dependent variables. Pearson's Chi-square test was used to compare categorical variables. Multivariable logistic regression was used to determine the association between immunotherapy usage and demographic characteristics and clinical variables. Median survival was analyzed using the Kaplan-Meier estimate and compared by the log-rank test. Multivariable Cox proportional hazards regression models were used to calculate hazard ratios (HRs) and $95 \%$ confidence intervals $(95 \% \mathrm{Cl})$ for the association between the three treatment groups and survival, adjusting for sex, age, race (white, other), type of hospital (academic, other), insurance status (private, 
other), Charlson-Deyo comorbidity score $(0, \geq 1)$. The NCDB does not include survival data for patients diagnosed in 2016, thus these patients were not included in survival analyses. StataSE v16.0 (Statacorp LLC, College Station, TX) was used for statistical analyses and a p-value $<0.05$ was used to indicate statistical significance.

\section{Results}

Demographic and clinical data. A total of 59,289 patients were identified, of whom 58,947 (99.4\%) received CT and $342(0.6 \%$ ) received IT-CT (Table 1). Patients who received IT-CT were younger (64 vs 66 years, $p=0.002$ ), more likely to be white, and more likely to have private insurance compared to those who received CT. Patients who received IT-CT had fewer comorbidities than patients who received CT (Charlson-Deyo score $=0: 76.3 \%$ vs $68.9 \%, p=0.007$ ). The majority of patients in the IT-CT group were treated at academic hospitals ( $56.6 \%$ IT-CT vs $39.7 \%$ CT, $p<0.001$ ). Usage of multi-agent chemotherapy was similar between the groups. 
Table 1

Summary of demographic and clinical data among patients with metastatic PDAC (NCDB, 2004-2016)

\begin{tabular}{|c|c|c|c|}
\hline Characteristic & $\begin{array}{l}\text { IT-CT } \\
(n=342)\end{array}$ & $\begin{array}{l}\text { CT } \\
(n=58,947)\end{array}$ & $\mathbf{p}$ \\
\hline Age (years; median, range) & $64(21-86)$ & $66(18-90)$ & 0.002 \\
\hline Male & $187(54.7 \%)$ & $32,058(54.4 \%)$ & 0.913 \\
\hline Race/Ethnicity & & & 0.008 \\
\hline White & $278(81.3 \%)$ & $44,770(75.9 \%)$ & \\
\hline Black & $21(6.1 \%)$ & $6,960(11.8 \%)$ & \\
\hline Hispanic & $18(5.3 \%)$ & $2,465(4.2 \%)$ & \\
\hline Other & $25(7.3 \%)$ & $4,752(8.1 \%)$ & \\
\hline Primary Insurance Payor & & & 0.001 \\
\hline None & $4(1.2 \%)$ & $1,744(3.0 \%)$ & \\
\hline Private & $168(49.1 \%)$ & $23,236(39.4 \%)$ & \\
\hline Medicare & $140(40.9 \%)$ & $29,183(49.5 \%)$ & \\
\hline Medicaid, Other & $30(8.8 \%)$ & $4,784(8.1 \%)$ & \\
\hline Education: $<6.3 \%$ of population $w / o$ high school diploma & $113(33.5 \%)$ & $15,862(27.3 \%)$ & 0.031 \\
\hline Income: $\geq \$ 63,333$ annual household income & $151(45.1 \%)$ & $22,149(38.2 \%)$ & 0.001 \\
\hline Charlson-Deyo score $=0$ & $261(76.3 \%)$ & $40,639(68.9 \%)$ & 0.007 \\
\hline Facility type & & & $<0.001$ \\
\hline Community & $25(7.5 \%)$ & $4,744(8.1 \%)$ & \\
\hline Comp Community & $97(29.0 \%)$ & $22,899(39.3 \%)$ & \\
\hline Academic/Research & $189(56.6 \%)$ & $23,126(39.7 \%)$ & \\
\hline Integrated Network & $23(6.9 \%)$ & $7,537(12.9 \%)$ & \\
\hline \multirow[t]{2}{*}{ Distance to hospital (miles; median, IQR) } & 17.8 & 10.1 & $<0.001$ \\
\hline & $(6.7,46.6)$ & $(4.5,24.2)$ & \\
\hline Tumor size (mm; median, IQR) & $40(30,52)$ & $38(30,51)$ & 0.520 \\
\hline Multi-agent chemo & $228(66.7 \%)$ & $37,043(62.8 \%)$ & 0.144 \\
\hline Single-agent chemo & $114(33.3 \%)$ & $21,904(37.2 \%)$ & \\
\hline
\end{tabular}


Factors associated with utilization of immunotherapy. Multivariable logistic regression analysis was used to determine what factors were associated with the administration of IT (Table 2). Advanced age and greater comorbidity score were associated with a decreased likelihood of receiving IT. Treatment at an academic facility was associated with an increased likelihood of receiving IT (OR $=1.95,95 \% \mathrm{Cl} 1.58-$ 2.41). There was no association between receipt of IT and sex, race, or insurance payor.

Table 2

Multivariable logistic regression analyzing utilization of immunotherapy among patients with metastatic PDAC (NCDB, 2004-2016).

\begin{tabular}{|lcccc|}
\hline & OR & $95 \%$ Cl & p-value \\
\hline Age & 0.99 & 0.98 & 1.00 & 0.046 \\
\hline Female vs male & 1.01 & 0.82 & 1.25 & 0.929 \\
\hline White vs other race & 1.30 & 0.99 & 1.71 & 0.055 \\
\hline Private insurance vs other & 1.23 & 0.96 & 1.56 & 0.100 \\
\hline Top 2 income quartiles vs bottom & 0.86 & 0.66 & 1.12 & 0.277 \\
\hline Top 2 education quartiles vs bottom & 1.34 & 1.03 & 1.74 & $\mathbf{0 . 0 3 1}$ \\
\hline Academic facility vs other & 1.95 & 1.58 & 2.41 & $<0.001$ \\
\hline Charlson-Deyo score $\geq 1$ vs 0 & 0.77 & 0.60 & 0.99 & $\mathbf{0 . 0 4 0}$ \\
\hline
\end{tabular}

Survival analyses. Patients who received combined IT-CT had longer median overall survival than patients treated with CT alone (7.9 vs 6.3 months, $p=0.005$; Table 3, Fig. 2). The two-year survival rates were similar (IT-CT: $7.32 \%$, CT: $7.01 \%$ ). Of note, there was an additional group of patients who received immunotherapy without chemotherapy. This group $(n=17)$ had a median survival of 5.2 months.

Table 3

Summary of median survival among patients with metastatic PDAC stratified by treatment group (NCDB, 2004-2015).

\begin{tabular}{|llllll|}
\hline Treatment & $\mathbf{n}$ & Median & IQR & & $\mathbf{p}$ \\
\hline IT-CT & 268 & 7.9 & 4.8 & 13.1 & 0.005 \\
CT & 52,344 & 6.3 & 3.2 & 11.7 & \\
IT only & 17 & 5.2 & 2.7 & 15.5 & - \\
\hline
\end{tabular}

Additionally, we performed a subgroup analysis for patients diagnosed in 2010 or later as both FOLFIRINOX and gemcitabine/nab-paclitaxel were introduced around that time. [2, 3] We found that the median survival of patients who received IT-CT increased to 9.7 months and was longer than those who received CT only (6.8 months, $p=0.017$, Table 4 , Fig. 3 ) 
Table 4

Summary of median survival among patients with metastatic PDAC stratified by treatment group (NCDB, 2010-2015).

\begin{tabular}{|llllll|}
\hline Treatment & $\mathbf{n}$ & Median & IQR & p \\
\cline { 1 - 5 } IT-CT & 152 & 9.7 & 5.1 & 15.1 & 0.017 \\
\cline { 1 - 5 } CT & 32,666 & 6.8 & 3.3 & 12.9 & \\
\hline
\end{tabular}

A multivariable Cox regression analysis examining overall survival in the treatment groups is shown in Table 5. IT-CT was associated with a survival advantage relative to $\mathrm{CT}$ alone $(\mathrm{HR}=0.86,95 \% \mathrm{Cl} 0.76-$ 0.97). Patients with advanced age, those treated at non-academic facilities, and those with a greater Charlson-Deyo score also had an associated survival disadvantage. Those who received multi-agent chemotherapy had an associated survival benefit $(\mathrm{HR}=0.65,95 \% \mathrm{Cl} 0.64-0.66)$.

Table 5

Multivariable Cox proportional hazards regression analyzing overall survival between treatment groups (NCDB, 2004-2015).

\begin{tabular}{|lllll|}
\hline & HR & $95 \%$ Cl & p-value \\
\hline IT-CT vs CT & 0.86 & 0.76 & 0.97 & 0.015 \\
\hline Age & 1.01 & 1.00 & 1.01 & $<0.001$ \\
\hline Female vs male & 0.93 & 0.91 & 0.95 & $<0.001$ \\
\hline Academic facility vs other & 0.83 & 0.82 & 0.85 & $<0.001$ \\
\hline Private insurance vs other & 0.94 & 0.92 & 0.96 & $<0.001$ \\
\hline Charlson-Deyo score $\geq 1$ vs 0 & 1.13 & 1.11 & 1.15 & $<0.001$ \\
\hline Multi-agent chemotherapy vs single-agent & 0.65 & 0.64 & 0.66 & $<0.001$ \\
\hline
\end{tabular}

\section{Discussion}

In recent years, immunotherapy has revolutionized the field of oncology. Several malignancies in which immunotherapy has improved survival, such as melanoma, lung, and renal cell carcinoma, are wellvascularized, highly immunogenic, have a high mutational burden, and have a microenvironment conducive to immune cell survival and function.[24, 25] Despite encouraging results in other malignancies, immunotherapy has not demonstrated efficacy in the majority of PDAC patients. More recently, olaparib (a poly (adenosine diphosphate-ribose) polymerase (PARP) inhibitor) was approved for utilization in patients with metastatic pancreatic cancer. There was no difference in median survival as compared to placebo (18.9 months vs 18.1 months), but olaparib was associated with an greater progression-free survival (7.4 months vs 3.8 months).[26] Of note, olaparib was only approved for patients with BRCA (breast cancer gene)-mutated pancreatic cancer, which accounts for just $5 \%$ of cases. 
[27] The median survival of both arms in the aforementioned study were greater than the current analysis. To be included in this trial, patients had to complete 16 weeks of chemotherapy, likely preventing enrollment of patients with poor performance status or rapidly progressive disease. Therefore, these results are not broadly generalizable to the majority of patients with metastatic PDAC.

In our study, we report that the addition of immunotherapy to chemotherapy was associated with a survival advantage in patients with metastatic PDAC. Median overall survival was approximately 1.6 months longer in the IT-CT group. This is concordant with a recent report which demonstrated that the combination of immunotherapy and chemotherapy in the adjuvant setting was associated with improved survival compared to adjuvant chemotherapy alone (5-year overall survival: $30.3 \%$ vs $20.6 \%$ ).[22] The associated survival advantage among patients who received adjuvant IT-CT as compared to adjuvant CT was greater in magnitude relative to the findings in our study. We hypothesize that this discrepancy may be due to the effectiveness of treatments based on disease burden. For example, when compared to single-agent gemcitabine, FOLFIRINOX lead to a 19.4 month survival advantage in the adjuvant setting, but just a 4.3 month advantage in metastatic PDAC. $[2,5]$ It is possible that our current findings, as well as those reported in the adjuvant setting, are false positive results, attributable to randomness in a relatively small sample size or unappreciated confounders.

Numerous immune targets have been studied in PDAC. A phase II trial in patients with metastatic PDAC who failed first-line chemotherapy received durvalumab, a monoclonal antibody targeting PD-L1 (programmed death-ligand 1); however, this trial was terminated due to a response rate of $0 \%$.[18] Cytotoxic T-lymphocyte-associated protein-4 (CTLA-4), another immune checkpoint, has been shown to downregulate immune responses. A single-arm clinical trial which combined ipilimumab (a CTLA-4 antagonist), nivolumab (a PD-1 (programmed cell death protein 1) antagonist), and radiation in patients with metastatic PDAC demonstrated an objective response rate of just $14 \%$ and a progression-free survival of 76 days.[19] A recent phase I trial examining the safety of nivolumab, nab-paclitaxel, and gemcitabine in patients with advanced PDAC did not show a survival benefit as compared to historic survival data without nivolumab.[28]

Deficiencies in mismatch repair with subsequent microsatellite instability (MSI) are often found in cancers and cause numerous genetic mutations, activating a patient's anti-tumor immune response.[29] A pivotal trial administered pembrolizumab (a PD-1 antagonist) to patients with metastatic cancer and demonstrated a disease control rate of $75 \%$ and a reported objective response rate of $62 \%$ in those with pancreatic cancer.[30] A follow-up trial, which also administered pembrolizumab to MSI-high/mismatch repair-deficient advanced cancers, reported an objective response rate in tumors of pancreatic-origin of just $18 \%$ with progression-free and overall survival of two and four months, respectively.[17] Importantly, only 1-2\% of PDAC tumors are MSI-high and/or mismatch repair-deficient.[31] It should be emphasized that the impact of immunotherapy on PDAC with MSI remains unproven, and the incidence of these tumors is extremely rare. One potential explanation for the discrepancy in effectiveness of immunotherapy relative to other cancers is due to the austere tumor microenvironment of PDAC, which includes a dense stromal compartment, low nutrient concentrations, and relative hypoxia.[15, 32] 
Patients' immune cells, which immunotherapies rely upon, are likely less effective under these harsh conditions, since they are not well adapted to survive in this harsh environment.[32, 33] A recent study by our group demonstrated that among all active phase III trials in the United States, $37 \%$ targeted the immune system.[34] While our data show that some patients benefit from immunotherapy, the two-year survival rate was just $7 \%$. Thus, ongoing research efforts may want to investigate other potential targets.

Our study also highlights disparities in utilization of immunotherapy. We found that advanced age and greater comorbidity index were associated with a decreased utilization of IT. Higher level education and receiving treatment at an academic center were associated with increased IT utilization. Disparities in cancer are well-studied and previous reports have shown variations in therapies administered and survival based on race and socioeconomic status.[35, 36] Studies have recommended multiple strategies, including insurance reform, improved access to quality care with community outreach, improved access to novel therapies, increased emphasis on prevention and screening, and diversification of employees in the healthcare field, to reduce disparities in cancer care.[37-39] Previous reports identified that Black and Asian patients, those who live alone, were unmarried or did not have children, had government issued insurance, or had issues performing their activities of daily living were more likely to decline all treatment. $[40,41]$

Although our study includes data on a large number of PDAC patients, there are limitations. Importantly, the use of immunotherapy is not common therefore the IT-CT treatment group is small in comparison to those who received CT alone. Based on limitations inherent to NCDB studies, including the lack of granularity of the data, we cannot comment on specific treatment details, such as the type of chemotherapy or immunotherapy received, whether the immunotherapy was part of a clinical trial, how many cycles of chemotherapy were completed, the genetic make-up of a patient's tumor (BRCA mutation, MSI status, etc.), and why immunotherapy was administered (progression of disease, patient desire, clinical trial, etc.). Also, the NCDB only includes data on the first six months of treatment, therefore a subset of patients in the CT group may have received IT later in their treatment course. Details of a patient's performance status are not recorded in the NCDB, thus Charlson-Deyo scores were used as a surrogate. The NCDB only receives data from Commission on Cancer-accredited hospitals and only captures $70 \%$ of cancer diagnoses. Therefore, these data cannot truly be generalized to all hospital systems and patients. We also cannot account for incorrect data within the database. Despite these limitations, our study reports a thorough analysis of the utilization of immunotherapy in patients with metastatic PDAC and demonstrates there may be a subset of patients who respond to immunotherapy.

\section{Conclusions}

In patients with metastatic PDAC, the addition of immunotherapy to chemotherapy was associated with improved median survival but a similar 2-year survival rate. Future research is needed to identify strategies that both stimulate entry of anti-tumor immune cells into the PDAC microenvironment and increase the anti-tumor capabilities of these cells. 


\section{Abbreviations}

BRCA

breast cancer gene

CT

chemotherapy

CTLA-4

cytotoxic T-lymphocyte-associated protein-4

FOLFIRINOX

5-fluorouracil, leucovorin, irinotecan, oxaliplatin

IT

immunotherapy

MSI

microsatellite instability

NCDB

National Cancer Database

PARP

poly (adenosine diphosphate-ribose) polymerase

PDAC

pancreatic ductal adenocarcinoma

PD-1

programmed cell death protein 1

PD-L1

programmed death-ligand 1

\section{Declarations}

- Ethics approval and consent to participate: Data within the National Cancer Database is deidentified therefore consent could not be obtained. The Institutional Review Boards at University Hospitals Cleveland Medical Center and Atrium Health have deemed that retrospective analyses of public, anonymized data sets are exempt from review. A waiver for consent was not required for this study.

- Consent for publication: Not applicable

- Availability of data and materials: The datasets during and/or analyzed during the current study available from the corresponding author on reasonable request

- Competing interests: None

- Funding: None

- Authors' contributions

- JJH - conceptualization, data curation, formal analysis, writing - original draft, writing - review and editing 
$\circ \mathrm{KB}$ - writing - review and editing

$\circ$ KS - writing - review and editing

- SCM - formal analysis, writing - review and editing

- LDR - writing - review and editing

- JMH - writing - review and editing

- JBA - writing - review and editing

- JMW - conceptualization, supervision, writing - review and editing

- LMO - supervision, writing - original draft, writing - review and editing

- Acknowledgements: none

\section{References}

1. NCCN guidelines version. 1.2019 pancreatic adenocarcinoma. National Comprehensive Cancer Network. https://www.nccn.org/professionals/physician_gls/PDF/pancreatic.pdf. Accessed 9 Mar 2020.

2. Conroy T, Desseigne F, Ychou M, Bouché O, Guimbaud R, Bécouarn $Y$, et al. FOLFIRINOX versus gemcitabine for metastatic pancreatic cancer. N Engl J Med. 2011;364:1817-25.

3. Von Hoff DD, Ervin T, Arena FP, Chiorean EG, Infante J, Moore M, et al. Increased Survival in Pancreatic Cancer with nab-Paclitaxel plus Gemcitabine. N Engl J Med. 2013;369:1691-703.

4. Cunningham D, Chau I, Stocken DD, Valle JW, Smith D, Steward W, et al. Phase III randomized comparison of gemcitabine versus gemcitabine plus capecitabine in patients with advanced pancreatic cancer. J Clin Oncol. 2009;27:5513-8.

5. Conroy T, Hammel P, Hebbar M, Ben Abdelghani M, Wei AC, Raoul J-L, et al. FOLFIRINOX or Gemcitabine as Adjuvant Therapy for Pancreatic Cancer. N Engl J Med. 2018;379:2395-406. doi:10.1056/NEJMoa1809775.

6. Powles T, Eder JP, Fine GD, Braiteh FS, Loriot Y, Cruz C, et al. MPDL3280A (anti-PD-L1) treatment leads to clinical activity in metastatic bladder cancer. Nature. 2014;515:558-64.

7. Choueiri TK, Xie W, Kollmannsberger C, North S, Knox JJ, Lampard JG, et al. The impact of cytoreductive nephrectomy on survival of patients with metastatic renal cell carcinoma receiving vascular endothelial growth factor targeted therapy. J Urol. 2011;185:60-6.

8. Dobry AS, Zogg CK, Hodi FS, Smith TR, Ott PA, lorgulescu JB. Management of metastatic melanoma: improved survival in a national cohort following the approvals of checkpoint blockade immunotherapies and targeted therapies. Cancer Immunol Immunother. 2018;67:1833-44.

9. Rizvi NA, Mazières J, Planchard D, Stinchcombe TE, Dy GK, Antonia SJ, et al. Activity and safety of nivolumab, an anti-PD-1 immune checkpoint inhibitor, for patients with advanced, refractory squamous non-small-cell lung cancer (CheckMate 063): A phase 2, single-arm trial. Lancet Oncol. 2015;16:257-65. 
10. Immunotherapy to treat cancer. National Cancer Institute. https://www.cancer.gov/aboutcancer/treatment/types/immunotherapy. Accessed 9 Mar 2020.

11. Manji GA, Olive KP, Saenger YM, Oberstein P. Current and emerging therapies in metastatic pancreatic cancer. Clin Cancer Res. 2017;23:1670-8.

12. Kunk PR, Bauer TW, Slingluff CL, Rahma OE. From bench to bedside a comprehensive review of pancreatic cancer immunotherapy. J Immunother Cancer. 2016;4:1-12. doi:10.1186/s40425-0160119-z.

13. Torphy RJ, Zhu Y, Schulick RD. Immunotherapy for pancreatic cancer: Barriers and breakthroughs. Ann Gastroenterol Surg. 2018;2:274-81.

14. Guo S, Contratto M, Miller G, Leichman L, Wu J. Immunotherapy in pancreatic cancer: Unleash its potential through novel combinations. World J Clin Oncol. 2017;8:230-40.

15. Looi CK, Chung FFL, Leong CO, Wong SF, Rosli R, Mai CW. Therapeutic challenges and current immunomodulatory strategies in targeting the immunosuppressive pancreatic tumor microenvironment. J Exp Clin Cancer Res. 2019;38:1-23.

16. Hilmi M, Bartholin L, Neuzillet C. Immune therapies in pancreatic ductal adenocarcinoma: Where are we now? World J Gastroenterol. 2018;24:2137-51. doi:10.3748/wjg.v24.i20.2137.

17. Marabelle A, Le DT, Ascierto PA, Di Giacomo AM, De Jesus-Acosta A, Delord J-P, et al. Efficacy of Pembrolizumab in Patients With Noncolorectal High Microsatellite Instability/Mismatch RepairDeficient Cancer: Results From the Phase II KEYNOTE-158 Study. J Clin Oncol. 2019;38:1-10. doi:10.1200/JC0.19.02105.

18. O'Reilly EM, Oh D-Y, Dhani N, Renouf DJ, Lee MA, Sun W, et al. Durvalumab With or Without Tremelimumab for Patients With Metastatic Pancreatic Ductal Adenocarcinoma: A Phase 2 Randomized Clinical Trial. JAMA Oncol. 2019;5:1431-8. doi:10.1001/jamaoncol.2019.1588.

19. Parikh A, Wo JY-L, Ryan DP, Clark JW, Nipp RD, Blaszkowsky LS, et al. A phase Il study of ipilimumab and nivolumab with radiation in metastatic pancreatic adenocarcinoma. J Clin Oncol. 2019;37 4_suppl:391. doi:10.1200/JC0.2019.37.4_suppl.391.

20. Brahmer JR, Tykodi SS, Chow LQM, Hwu W-J, Topalian SL, Hwu P, et al. Safety and Activity of AntiPD-L1 Antibody in Patients with Advanced Cancer. N Engl J Med. 2012;366:2455-65.

21. 10.1158/1078-0432.CCR-20-0099.

22. Tran TB, Maker VK, Maker AV. Impact of Immunotherapy after Resection of Pancreatic Cancer. J Am Coll Surg. 2019;229:19-27.e1. doi:10.1016/j.jamcollsurg.2019.01.016.

23. American College of Surgeons. The National Cancer Database 2016 PUF Data Dictionary.

24. Lugowska I, Teterycz P, Rutkowski P. Immunotherapy of melanoma. Contemp Oncol. 2018;22:61-7. doi:10.5114/wo.2018.73889.

25. Santoni M, Massari F, Di Nunno V, Conti A, Cimadamore A, Scarpelli M, et al. Immunotherapy in renal cell carcinoma: latest evidence and clinical implications. Drugs Context. 2018;7:212528. doi:10.7573/dic.212528. 
26. Golan T, Hammel P, Reni M, Van Cutsem E, Macarulla T, Hall MJ, et al. Maintenance Olaparib for Germline BRCA -Mutated Metastatic Pancreatic Cancer. N Engl J Med. 2019;381:317-27.

27. Holter S, Borgida A, Dodd A, Grant R, Semotiuk K, Hedley D, et al. Germline BRCA mutations in a large clinic-based cohort of patients with pancreatic adenocarcinoma. J Clin Onc. 2015;33:3124-9.

28. Wainberg ZA, Hochster HS, Kim EJ, George B, Kaylan A, Chiorean EG, et al. Open-label, Phase I Study of Nivolumab Combined with nab -Paclitaxel Plus Gemcitabine in Advanced Pancreatic Cancer. Clin Cancer Res. 2020;26.

29. Zhao P, Li L, Jiang X, Li Q. Mismatch repair deficiency/microsatellite instability-high as a predictor for anti-PD-1/PD-L1 immunotherapy efficacy. J Hematol Oncol. 2019;12:54. doi:10.1186/s13045-0190738-1.

30. Le DT, Durham JN, Smith KN, Wang H, Bartlett BR, Aulakh LK, et al. Mismatch repair deficiency predicts response of solid tumors to PD-1 blockade. Science. 2017;357:409-13. doi:10.1126/science.aan6733.

31. Ahmad-Nielsen SA, Bruun Nielsen MF, Mortensen MB, Detlefsen S. Frequency of mismatch repair deficiency in pancreatic ductal adenocarcinoma. Pathol Res Pract. 2020;216:152985. doi:10.1016/j.prp.2020.152985.

32. Vaziri-Gohar A, Zarei M, Brody JR, Winter JM. Metabolic Dependencies in Pancreatic Cancer. Front Oncol. 2018;8:617. doi:10.3389/fonc.2018.00617.

33. Bonaventura P, Shekarian T, Alcazer V, Valladeau-Guilemond J, Valsesia-Wittmann S, Amigorena S, et al. Cold Tumors: A Therapeutic Challenge for Immunotherapy. Front Immunol. 2019;10:168. doi:10.3389/fimmu.2019.00168.

34. Katayama E, Hue JJ, Bajor DL, Ocuin LM, Ammori JB, Hardacre JM, et al. A comprehensive analysis of clinical trials in pancreatic cancer: what is coming down the pike? Oncotarget. 2020;11:3489-501.

35. O'Keefe EB, Meltzer JP, Bethea TN. Health disparities and cancer: racial disparities in cancer mortality in the United States, 2000-2010. Front public Heal. 2015;3:51. doi:10.3389/fpubh.2015.00051.

36. Ward E, Jemal A, Cokkinides V, Singh GK, Cardinez C, Ghafoor A, et al. Cancer Disparities by Race/Ethnicity and Socioeconomic Status. CA Cancer J Clin. 2004;54:78-93.

37. Chin $\mathrm{MH}$, Walters $\mathrm{AE}$, Cook SC, Huang ES. Interventions to reduce racial and ethnic disparities in health care. Med Care Res Rev. 2007;64 5_Suppl:7-28.

38. Kagawa-Singer M, Valdez Dadia A, Yu MC, Surbone A. Cancer, Culture, and Health Disparities: Time to Chart a New Course? CA Cancer J Clin. 2010;60:12-39.

39. Moy B, Polite BN, Halpern MT, Stranne SK, Winer EP, Wollins DS, et al. American Society of Clinical Oncology policy statement: Opportunities in the patient protection and affordable care act to reduce cancer care disparities. J Clin Onc. 2011;29:3816-24.

40. Puts MTE, Monette J, Girre V, Wolfson C, Monette M, Batist G, et al. Characteristics of older newly diagnosed cancer patients refusing cancer treatments. Support Care Cancer. 2010;18:969-74. 
41. Restrepo DJ, Sisti A, Boczar D, Huayllani MT, Fishe J, Gabriel E, McLaughlin SA, Bagaria S, Spaulding A, Rinker BDFA. Characteristics of Breast Cancer Patients Who Refuse Surgery. Anticancer Res. 2019;39:4941-5.

\section{Figures}

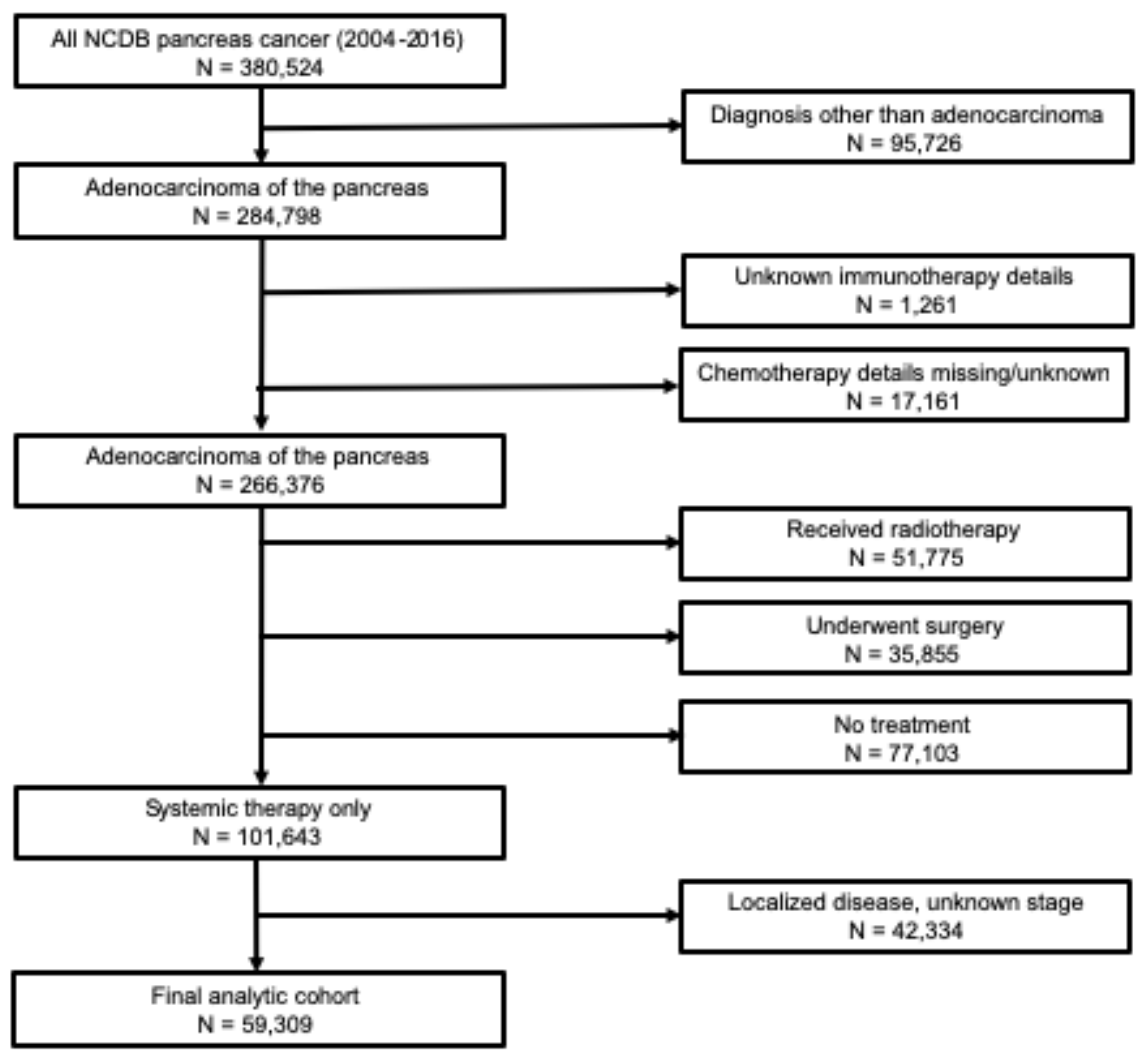

Figure 1

Consort diagram of study population 


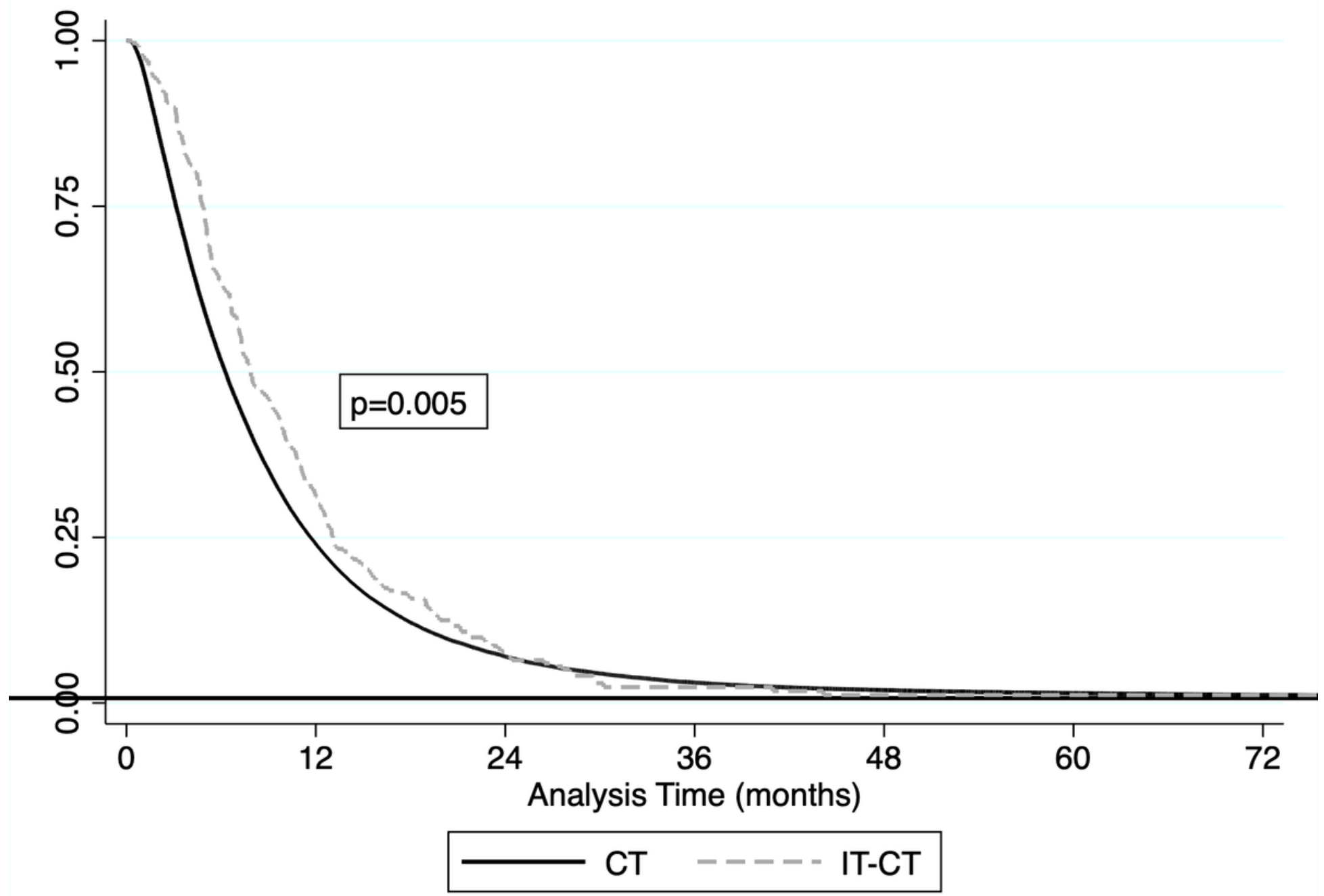

Figure 2

Kaplan-Meier estimates of survival by treatment group (NCDB, 2004-2015). 


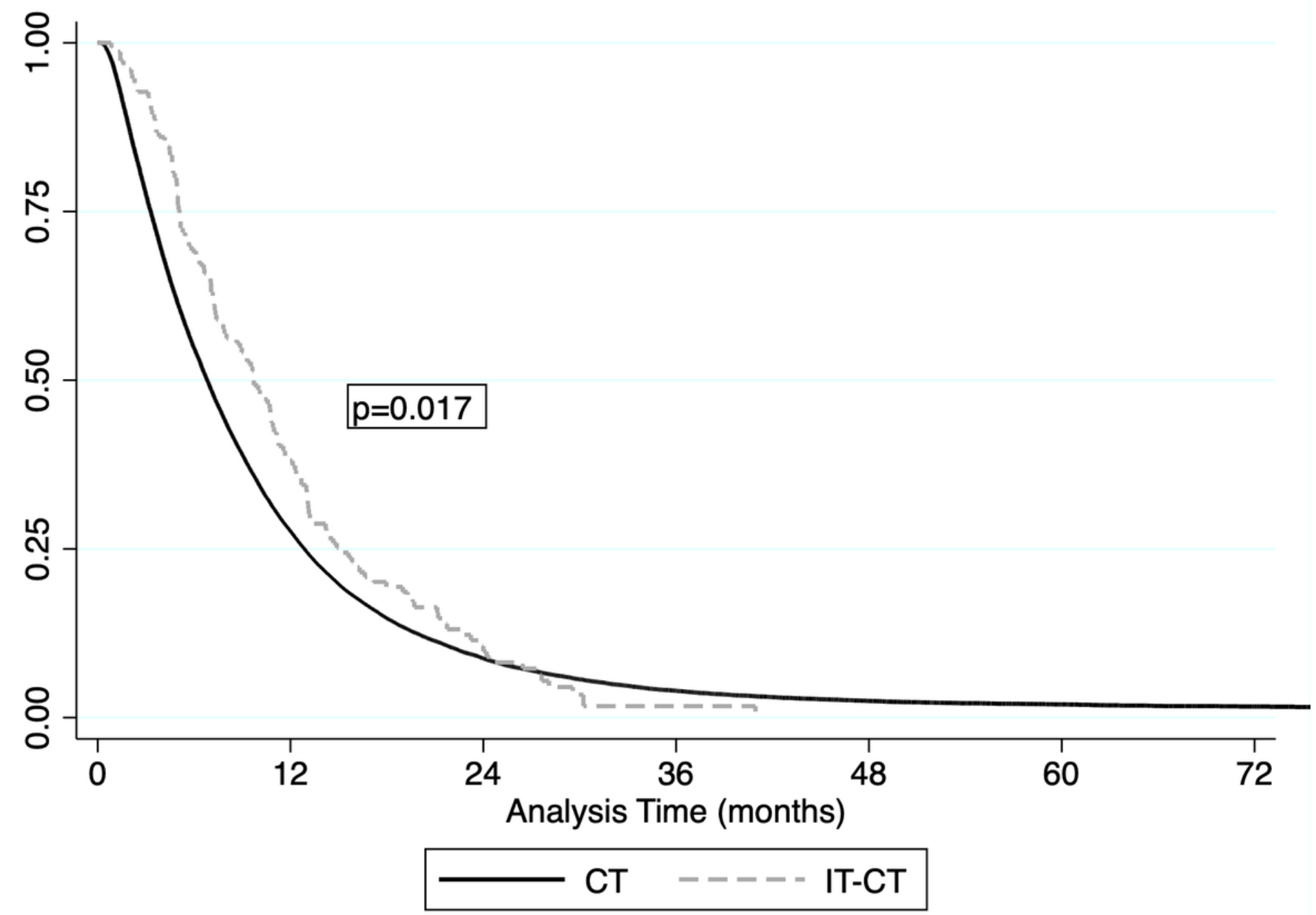

Figure 3

Kaplan-Meier estimates of survival by treatment group (NCDB, 2010-2015). 